\title{
Quality of life after primary septorhinoplasty in deviated- and non-deviated nose measured with ROE, FROI-17 and
} SF-36*

\author{
O.C. Bulut', F. Wallner', R. Hohenberger', P.K. Plinkert', I. Baumann' \\ Rhinology 55: 75-80, 2017 \\ Department of Otolaryngology, University of Heidelberg, Germany \\ *Received for publication: \\ August 23, 2016 \\ Accepted: October 2, 2016
}

https:://doi.org/10.4193/Rhino16.243

\begin{abstract}
Background: Quality of life measurements are gaining in importance. The present study was conducted with the aim to compare patient satisfaction after septorhinoplasty according to their preoperative nasal deformity.

Methods: The patients completed two disease-specific questionnaires before their surgery: the Functional Rhinoplasty Outcome Inventory (FROI-17), the Rhinoplasty Outcome Evaluation (ROE) and as a general instrument, the Short Form 36 Health Survey (SF-36). The second measurement was taken during an outpatient examination 12 months after their primary septorhinoplasty. Patients were grouped in nasal axis deviation (NAD), nasal hump deformity (NHD) and NAD plus NHD. Additionally the patients with preoperative NAD and NAD+NHD were combined as "deviated nose" and compared to the "non-deviation group."

Results: One hundred and two patients (51 male and 51 female) underwent primary septorhinoplasty. The ROE- and the FROI-17 overall score including all three subgroups showed significant postoperative improvements. Regarding the SF-36, the postoperative score improved significantly only in two scales (role-functioning physical and mental health). Looking at the the different QoL questionnaires, there were significant postoperative differences in regards to deviated versus non-deviated nose in the FROI-17 overall- and FROI-17 subscores (nasal and general symptoms) and in three scales of the SF-36 (vitality, social functioning, rolefunctioning emotional), showing a greater postoperative satisfaction in the "deviated-nose patient."
\end{abstract}

Conclusion: Patients with and without nasal deviation showed improved QoL after their surgery, as measured with the ROE, the FROI-17 and the SF-36. The patients with a nasal deviation showed a significantly better outcome, as measured with the FROI-17, in comparison with the "non-deviated group."

Key words: Rhinoplasty, quality of life, deviated nose, FROI-17, ROE

\section{Introduction}

Quality of life measurements are gaining in importance. The aim of a septorhinoplasty is to increase patients' quality of life after surgery. For a long time the only validated disease-specific instrument was the Rhinoplasty Outcomes Evaluation (ROE) (1). The ROE focuses on the aesthetic aspects of septorhinoplasty. To evaluate functional and aesthetic aspects of septorhinoplasty the Functional Rhinoplasty Outcome Inventory 17 (FROI-17) was developed and validated ${ }^{(2)}$. The present study was conducted with the aim of comparing patient satisfaction after primary sep- torhinoplasty depending on their preoperative nasal deformity.

\section{Materials and methods}

The Ethics Commitee of the Medical Faculty at the University of Heidelberg granted permission to conduct the study (Project No. 409/2006). Informed consent was obtained from all patients. The patients were evaluated during an outpatient examination usually one day before the surgery. They were asked to fill out questionnaires including two disease-specific questionnaires, the Functional Rhinoplasty Outcome Inventory (FROI-17), 
the Rhinoplasty Outcome Evaluation (ROE) and as a general instrument, the Short Form 36 Health Survey (SF-36). The second measurement was taken during an outpatient examination 12 months after the primary septorhinoplasty.

Regarding the disease-specific questionnaires, the ROE contains six items. Five of the six items are related solely to aesthetic aspects and only one item has a functional alignment. Each response can be graded between zero and four. The total score may therefore vary between zero and 24 . The score is then transferred into a 0-100 scale (divided by 24 and multiplied by 100 ). The higher the score, the greater the satisfaction of the patient regarding his or her septorhinoplasty.

The second disease-specific questionnaire, the FROI-17, detects more functional aspects than the ROE ${ }^{(3)}$ and includes 17 items, which can be graded between zero (no problem) and five (as bad as it can be). The overall score is then transformed to a 0-100 scale by dividing the sum of the raw scores of the items by the sum of ranges of the items followed by a multiplication by 100 .

The SF-36 Health Survey consists of 36 items, grouped into eight aspects: physical functioning, role-functioning physical, bodily pain, general health, vitality, social functioning, role-functioning emotional and mental health. Rules for item scoring and scales are available in the SF-36 scoring manual. Higher scores indicate a more positive rating.

General information (age, gender, allergies, medication, medical and surgical history) from all patients was obtained. Patients were classified preoperatively by the surgeon into three groups according to their nasal deformity: nasal hump deformity (NHD), nasal axis deviation (NAD) and NAD + NHD. Additionally the patients with preoperatively NAD and NAD+NHD were combined as "deviated nose" and compared to the "non-deviated" group (NHD). All patients in this study underwent primary septorhinoplasty.

The statistical analysis was performed using the statistical software JMP version 12.0.0. (SAS Institute Inc., Cary, NC, USA). Testing for significant differences between two groups of patients was performed using Wilcoxon-test (nonparametric). The significance level was set at $\mathrm{p}<0.05$. Moreover, the pre- and postoperative scores of the ROE, the FROI-17 and SF-36 were calculated.

\section{Results}

One hundred and two patients (51 male and 51 female) underwent primary septorhinoplasty and were operated by two of the authors (F.W. and I.B.). Our response rate was $67 \%$. The patients were $28.7 \pm 11.4$ years old. They were classified into three groups: NHD (24 patients), NAD (28 patients) and NAD + NHD
(50 patients).

The ROE overall score across NHD, NAD and NHD+NAD increased from 42.2 ( \pm 15.7 ) preoperatively to 63.9 ( \pm 18.9$), p=0.0001$, postoperatively, indicating a greater satisfaction postoperatively.

The FROI-17 across NHD, NAD and NHD+NAD showed significant postoperative improvement of subjective assessments by the patients. The overall score decreased from 32.5 ( \pm 18.2$)$ preoperatively to 20.3 ( \pm 18.4$), p=0.0001$, postoperatively. All subscores (nasal symptoms preoperatively $32.4( \pm 16.1)$ to $20.9( \pm 19.3)$, $\mathrm{p}=0.0002$, postoperatively; general symptoms preoperatively $32.8( \pm 24.8)$ to $20.6( \pm 21.5), p=0.003$, postoperatively and self confidence $32.2( \pm 27.5)$ preoperatively to $17.1( \pm 21.8), p=0.0005$, postoperatively) showed significant improvement. Lower scores indicate greater patient satisfaction in this questionnaire.

Regarding the SF-36, the postoperative score improved significantly only in two scales (1. role-functioning physical: preoperatively $75.4( \pm 35.5)$ to postoperatively $90.9( \pm 22.3), p=0.003 ; 2$. mental health: preoperatively $62.6( \pm 19.9)$ to postoperatively $69.2( \pm 17.7), p=0.04)$.

Regarding the different preoperative nasal deformities (NHD, $\mathrm{NAD}$ and NHD+NAD), there was an improvement in all three subgroups measured with the ROE and the FROI-17 (Table 1).

In our study an influence of postoperative $\mathrm{QOL}$ in regards to open versus closed approach (closed approach $72.8 \%, \mathrm{n}=75$; open approach $27.2 \%, \mathrm{n}=28$ ) was not detectable.

To analyze the impact of a deviated nose on QoL, the NAD and $\mathrm{NAD}+\mathrm{NHD}$ were grouped together and compared with the scores of septorhinoplasty patients without a nasal deviation.

Both, the deviated- and the non-deviated nose patients improved significantly after septorhinoplasty measured with the ROE and the FROI-17. There were no significant differences in the overall ROE score of deviated versus non-deviated noses preoperatively or postoperatively. There were significant differences preoperatively and postoperatively in the FROI-17 overall scores and in the subcategory "nasal symptoms." The patients with a deviated nose had lower overall- and "nasal symptom" scores before and after surgery. Significant differences were found in the "general symptom" postoperative scores of the FROI-17, showing lower scores in the deviated nose patients (Table 2), indicating higher patient satisfaction.

Regarding the SF-36 significant differences were found between the preoperative scores in the scale "physical functioning", with higher scores in the deviated-nose patient. 
Table 1. Preoperative versus postoperative scores.

\begin{tabular}{|lccc|}
\hline & preoperative & postoperative & p-value \\
\hline ROE overall score & & & \\
\hline NHD & $41.3 \pm 12$ & $64.4 \pm 20.8$ & $\mathbf{0 . 0 4}$ \\
\hline NAD & $40.2 \pm 15.4$ & $65.2 \pm 18.7$ & $\mathbf{0 . 0 4}$ \\
\hline NHD+NAD & $43.3 . \pm 17$ & $63.2 \pm 18.9$ & $\mathbf{0 . 0 0 1}$ \\
\hline FROI-17 overall score & & & \\
\hline NHD & $42.8 \pm 18.1$ & $35.3 \pm 22.6$ & $\mathbf{0 . 0 4}$ \\
\hline NAD & $29.7 \pm 19.1$ & $18.8 \pm 16.7$ & $\mathbf{0 . 0 4}$ \\
\hline NHD+NAD & $30.9 \pm 17.2$ & $16.9 \pm 16.1$ & $\mathbf{0 . 0 0 3}$ \\
\hline
\end{tabular}

NHD: nasal hump deformity; NAD: nasal axis deviation

Significant differences between the postoperative scores were found in the scales "vitality", "social functioning", and "role-functioning emotional." The deviated nose patients showed significant higher scores in those scales (Table 2).

\section{Discussion}

Septorhinoplasty is among the most common surgeries performed by facial plastic surgeons worldwide ${ }^{(4)}$ and subjective evaluation of postoperative results with regard to patients' satisfaction is a growing challenge ${ }^{(5)}$. Different studies have shown postoperative improvements of the ROE- and FROI-17 score after rhinoplasty ${ }^{(3,6,7)}$. To our knowledge only three articles in the literature have described postoperative outcomes according to nasal deformity. All of these articles described their assessment results with the ROE ${ }^{(7-9)}$. As the ROE focuses mainly on the cosmetic outcome, we have added another validated disease-specific questionnaire the FROI-17, emphasizing both functional and aesthetic outcome of septorhinoplasty, as well as the non-rhinoplasty-specific instrument SF-36. This is the first study looking at quality of life outcome according to preoperative nasal deformity with the ROE, FROI-17 and SF-36.

Facial symmetry and proportions have been recognized as important determinants of attractiveness ${ }^{\left({ }^{(10)}\right.}$. Arima et al. ${ }^{\left({ }^{8}\right)}$ found that the mean ROE satisfaction score of patients who underwent rhinoplasty due to a crooked nose increased from $24.6 \pm 11.3$ to $76.1 \pm 19.5$ postoperatively, which is in line with our findings. In our cohort the ROE score in the nasal deviation group (NAD and NAD+NHD) increased from $41.3 \pm 12$ to $64.4 \pm 20.8$ after surgery. The FROI-17 decreased in both subgroups indicating an increase in disease specific QoL. In this study significant postoperative improvements regarding the SF-36, were found in the scales: role-functioning physical and mental health. An improved outcome after septorhinoplasty in the scale mental health was also found by Klassen et al. ${ }^{(11)}$. It can be argued that our cohort was predominantely young and healthy, and that therefore their mental impairment affected their physical conditions ${ }^{(3)}$.

It can be seen that all three subgroups (NHD, NAD and $\mathrm{NHD}+\mathrm{NAD}$ ) improved approximately equally in the diseasespecific quality of life questionnaires ROE and FROI-17 scores after surgery. The impairment in QoL measured with the ROE preoperatively is approximately equal in all nasal deformities. Looking at the preoperative FROI-17 scores, NHD deformity starts with a higher score than NAD and NHD+NAD, indicating a significant higher functional impairment preoperatively. As in our sample NHD was the least frequent preoperative deformity, this could be explained as a statistical abnormality due to our limited patients in this category.

In our sample, NAD and NAD+NHD was the dominant nasal deformity. Baykal et al. ${ }^{(9)}$ showed an increase in ROE score postoperatively according to different nasal deformities preoperatively. They showed that the ROE score in patients with NHD (from 21 to 89 ), NAD (from 25 to 72) and NHD+NAD (from 27 to 80 ) increased postoperatively, indicating higher patient satisfaction, which is in line with our findings.

Regarding the ROE we could not find a significant difference in QoL changes between our subgroups, probably due to a limited number of patients, which can be seen as a weakness of our study. Another reason for our findings could be our predominantly young collective with $28.7 \pm 11.4$ years in average. Arima et al. ${ }^{(8)}$ reported that patients younger than 30 years had lower satisfaction increases compared with patients over 30 years. We found significant differences in the overall FROI-17 score between the deviated and the non-deviated patients and in the subcategory "nasal symptoms," at the time pre- and postoperatively. The non-deviated patients showed, before and after their surgery, a higher nasal impairment and, compared to the patients with a deviation, a lower satisfaction regarding his or her septorhinoplasty. In the subgroup "general symptoms" the patients with a preoperatively deviated nose were more satisfied postoperatively than those without a deviation. Whereas the ROE focuses mainly on the aesthetic side of septorhinoplasty, the FROI-17 detects also the functional aspects of this surgery. As the deviated nose is usually the cause of a trauma, these patients experienced a nasal impairment shortly after their trauma and can therefore possibly compare between the pre-and postoperative results better, leading to a higher satisfaction in the FROI-17 subgroup "nasal problems" and also in the FROI-17 overall score. In addition, in our experience patients with NHD tend to be more critical of their postoperative outcome, leading in our opinion to a lower increase in the Qol questionnaire. Baykal ${ }^{(9)}$ reported a higher postoperative satisfaction in the deviated nose. Cingi et al. ${ }^{(12)}$ showed an increase in ROE score 
Table 2. Pre- and postoperative scores of FROI-17, ROE and SF-36 in regards to deviation vs. non-deviation nasal deformity.

\begin{tabular}{|c|c|c|c|c|c|}
\hline & \multicolumn{2}{|c|}{ Deviated } & \multicolumn{2}{|c|}{ Non-Deviated } & \multirow[b]{2}{*}{ p-value } \\
\hline & Mean & SD & Mean & SD & \\
\hline \multicolumn{6}{|c|}{ FROI-17 overall score } \\
\hline preop & 30,5 & 17,7 & 42,8 & 18,1 & 0,03 \\
\hline postop & 17,4 & 16,2 & 35,3 & 22,6 & 0,01 \\
\hline \multicolumn{6}{|c|}{ FROI-17 nasal symptoms } \\
\hline preop & 29,9 & 14,4 & 45,2 & 8,9 & 0,01 \\
\hline postop & 18,0 & 16,0 & 36,1 & 27,5 & 0,03 \\
\hline \multicolumn{6}{|c|}{ FROI-17 general symptoms } \\
\hline preop & 31,5 & 25,3 & 39,5 & 22,3 & 0,3 \\
\hline postop & 17,1 & 18,8 & 39,2 & 25,9 & 0,01 \\
\hline \multicolumn{6}{|c|}{ FROI-17 self confidence } \\
\hline preop & 29,5 & 26,1 & 46,4 & 31,4 & 0,1 \\
\hline postop & 16,7 & 22,6 & 19,1 & 18,1 & 0,7 \\
\hline \multicolumn{6}{|c|}{ ROE overall score } \\
\hline preop & 42,3 & 16,4 & 41,3 & 12,0 & 0,8 \\
\hline postop & 63,8 & 18,7 & 64,4 & 20,8 & 0,9 \\
\hline \multicolumn{6}{|l|}{ SF-36 } \\
\hline \multicolumn{6}{|c|}{ physical functioning } \\
\hline preop & 87,9 & 15,7 & 71,4 & 29,4 & 0,048 \\
\hline postop & 93,3 & 12,5 & 79,1 & 29,8 & 0,15 \\
\hline \multicolumn{6}{|c|}{ role-functioning physical } \\
\hline preop & 77,2 & 35,1 & 65,9 & 37,5 & 0,4 \\
\hline postop & 93,5 & 19,1 & 77,3 & 32,5 & 0,13 \\
\hline \multicolumn{6}{|c|}{ bodily pain } \\
\hline preop & 80,3 & 27,9 & 69,0 & 32,1 & 0,3 \\
\hline postop & 87,1 & 20,3 & 79,5 & 27,2 & 0,4 \\
\hline \multicolumn{6}{|c|}{ general health } \\
\hline preop & 68,2 & 21,5 & 62,0 & 27,6 & 0,5 \\
\hline postop & 71,4 & 20,0 & 63,1 & 25,7 & 0,3 \\
\hline \multicolumn{6}{|l|}{ vitaliy } \\
\hline preop & 53,9 & 19,8 & 47,3 & 23,8 & 0,4 \\
\hline postop & 59,1 & 17,3 & 45,5 & 20,4 & 0,03 \\
\hline \multicolumn{6}{|c|}{ social functioning } \\
\hline preop & 77,5 & 25,8 & 70,5 & 25,8 & 0,4 \\
\hline postop & 82,8 & 20,8 & 60,2 & 29,5 & 0,02 \\
\hline \multicolumn{6}{|c|}{ role-functioning emotional } \\
\hline preop & 83,6 & 30,3 & 69,7 & 37,9 & 0,3 \\
\hline postop & 89,7 & 25,9 & 63,6 & 43,3 & 0,04 \\
\hline \multicolumn{6}{|c|}{ mental health } \\
\hline preop & 64,1 & 19,5 & 54,5 & 20,9 & 0,2 \\
\hline postop & 70,1 & 16,1 & 64,7 & 25,1 & 0,5 \\
\hline
\end{tabular}

SD: standard deviation 
in patients with a deviated nose postoperatively, however the non-deviated patients showed a slightly better outcome. It was stated that the slight difference in patient satisfaction is likely due to personal perception and emotional status. In our opinion, the different results in our study are not a contradiction, but due to different QoL questionnaires, which also focuses on functional alignments.

Regarding the SF-36, patients with a deviated nose showed a significantly higher postoperative score indicating a more positive rating in the scales "vitality," "social functioning," and "role functioning emotional." An increase in SF-36 subscores after septorhinoplasty is reported in the literature ${ }^{(11)}$. In our predominantly young collective it is conceivable that patients with a deviated nose after trauma are more impaired in their emotional status and in their personal perception, resulting in a greater satisfaction for a non-deviated nose after their rhinoplasty.

The significant differences in the deviation versus non-deviation patients in the FROI-17 and three scales of the SF-36 indicate a stronger correlation of the FROI-17 with the SF-36, than the SF-36 with the ROE, which did not show significant differences in the overall score in regards to deviated versus non-deviated patients. Correlation analysis revealed that the correlations between the scales of the SF-36 and the FROI-17 were significantly more pronounced than the correlations between the SF36 and the ROE ${ }^{(3)}$, due to the fact that the $\mathrm{FROI}-17$ focuses also on the functional aspect of septorhinoplasty, which supports our findings.

Looking at the literature, there has been limited published data about the role of patient satisfaction according to the preoperative nasal deformity. To our knowledge this is the first study to examine QoL with three different questionnaires (two of them disease-specific) with a one-year follow-up showing an increase in patient satisfaction according to different preoperative nasal pathologies. The weakness of our study is the limited number of patients, a predominantly young sample and our main nasal deformity being the "deviated nose." Our study contributes to the thesis that rhinoplasty improves QoL in patients with and without a deviated nose. Further prospective studies with larger samples and with disease-specific QoL questionnaires, which concentrate on the functional aspect of septorhinoplasty are necessary to evaluate the degree of patient satisfaction in different deformities.

\section{Conclusion}

QoL improves significantly in patients with NHD, NAD and NHD+NAD after surgery.

The ROE score including all three subgroups increased, indicating a greater satisfaction after surgery. The FROI-17 including all subgroups showed significant postoperative improvement of subjective assessments by the patients. Regarding the SF-36, the postoperative score improved significantly only in two scales (role-functioning physical and mental health). Patients with and without nasal deviation showed improved QoL measured with the ROE and the FROI-17. Regarding the different QoL questionnaires, there were significant postoperative differences in deviated versus non-deviated nose patient, in the FROI-17 overall-, FROI-17 subscores (nasal and general symptoms) and in three scales of the SF-36 (vitality, social functioning, role-functioning emotional), indicating a greater postoperative satisfaction in the deviated-nose patient. We attribute this to the fact that the FROI-17 highlights the functional aspects of septorhinoplasty.

\section{Authorship contribution}

All authors made substantial contributions to the study. OCB: designed and coordinated the study, participated in the data acquisition and analysis, interpreted the data and drafted the manuscript.

FW: participated in the data acquisition and analysis, critically revised the manuscript for important intellectual content.

$\mathrm{RH}$ : participated in the data acquisition and analysis PKP: participated in the data acquisition and analysis, critically revised the manuscript for important intellectual content IB: designed and coordinated the study, participated in the data acquisition and analysis, interpreted the data, critically revised the manuscript for important intellectual content

\section{Conflict of interest}

None to declare.

\section{References}

1. Alsarraf R. Outcomes research in facial plastic surgery: a review and new directions. Aesth Plast Surg. 2000;24(3):192-7.

2. Bulut C, Wallner F, Plinkert PK, Baumann I. Development and validation of the Functional Rhinoplasty Outcome Inventory 17 (FROI-17). Rhinology. 2014;52(4):315-9.

3. Bulut OC, Wallner F, Plinkert PK, Prochnow S, Kuhnt C, Baumann I. Quality of life after septorhinoplasty measured with the Functional
Rhinoplasty Outcome Inventory 17 (FROI17). Rhinology. 2015;53(1):54-8.

4. Apaydin F. Rhinoplasty at the global crossroads. Arch Facial Plast Surg. 2009;11(6):4213.

5. Alsarraf R, Larrabee WF, Jr., Anderson S, Murakami CS, Johnson CM, Jr. Measuring cosmetic facial plastic surgery outcomes: a pilot study. Arch Facial Plast Surg. 2001;3(3):198-201

6. Saleh AM, Younes A, Friedman O. Cosmetics and function: quality-of-life changes after rhinoplasty surgery. Laryngoscope. 2012;122(2):254-9.

7. Cingi C, Songu M, Bal C. Outcomes research in rhinoplasty: body image and quality of life. Am J Rhinol Allergy. 2011;25(4):263-7.

8. Arima LM, Velasco LC, Tiago RS. Crooked nose: outcome evaluations in rhinoplasty. Braz J Otorhinolaryngol. 2011;77(4):510-5.

9. Baykal B, Erdim I, Kayhan FT, Oghan F. Comparative analysis of nasal deformities 
according to patient satisfaction. J Oral Maxillofac Surg. 2014;72(3):603 e1-7.

10. Chatrath P, De Cordova J, Nouraei SA, Ahmed J, Saleh HA. Objective assessment of facial asymmetry in rhinoplasty patients. Arch Facial Plast Surg. 2007;9(3):184-7.

11. Klassen A, Jenkinson C, Fitzpatrick R, Goodacre T. Patients' health related quality of life before and after aesthetic surgery. $\mathrm{Br}$ J Plast Surg. 1996;49(7):433-8.

12. Cingi C, Eskiizmir G. Deviated nose attenuates the degree of patient satisfaction and quality of life in rhinoplasty: a prospective controlled study. Clin Otolaryngol. 2013;38(2):136-41.
O.C. Bulut, MD

Department of Otolaryngology University Hospital Heidelberg Im Neuenheimer Feld 400 69120 Heidelberg Germany

Tel: +49 (0)6221-6705

Fax: +49 (0)6221-563 3967

E-mail:

cem.bulut@med.uni-heidelberg.de

\section{ADVERTISEMENT}

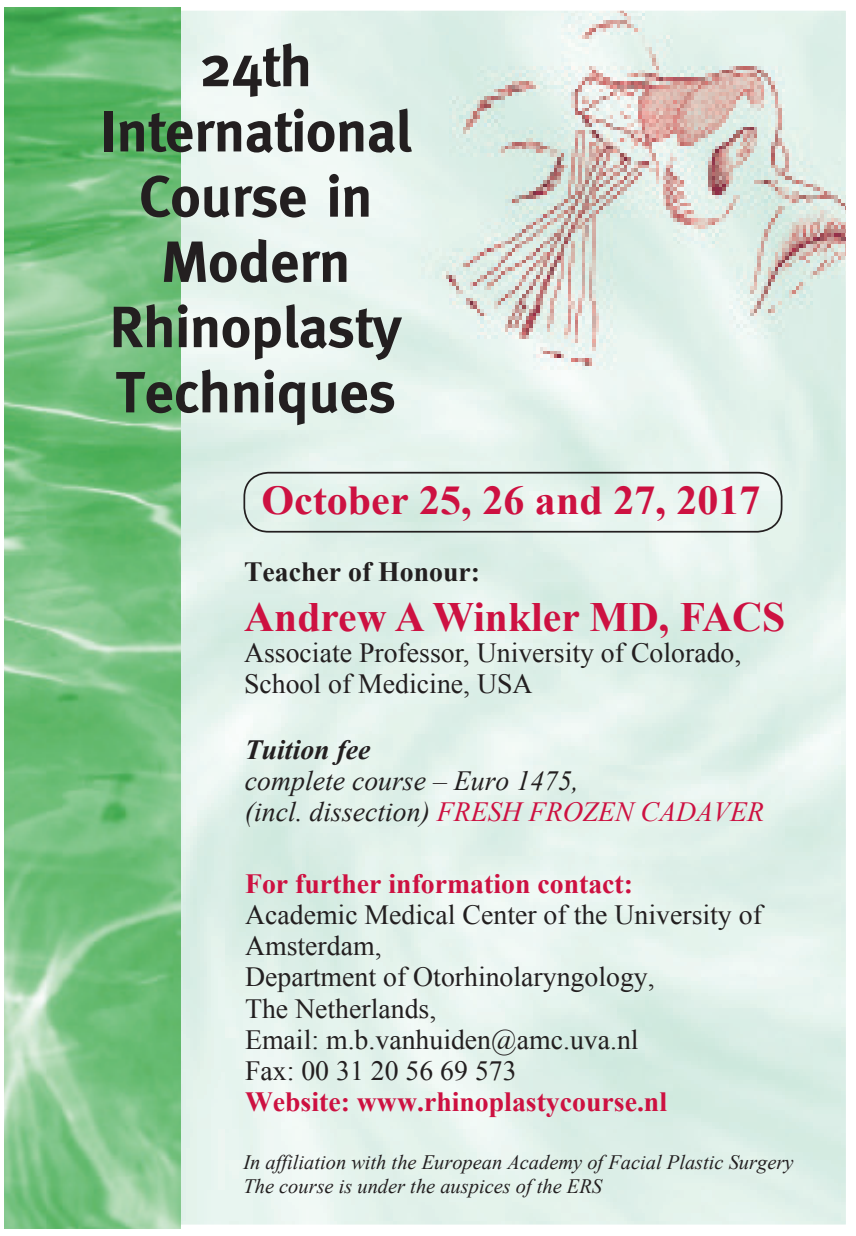

